AperTO - Archivio Istituzionale Open Access dell'Università di Torino

\title{
Use of eltrombopag in aplastic anemia in Europe
}

\section{This is a pre print version of the following article:}

Original Citation:

Availability:

This version is available http://hdl.handle.net/2318/1707454

since 2019-07-26T09:46:04Z

Published version:

DOI:10.1007/s00277-019-03652-8

Terms of use:

Open Access

Anyone can freely access the full text of works made available as "Open Access". Works made available under a Creative Commons license can be used according to the terms and conditions of said license. Use of all other works requires consent of the right holder (author or publisher) if not exempted from copyright protection by the applicable law. 


\title{
Use of eltrombopag in aplastic anemia in Europe
}

\author{
Matyas Ecsedi ${ }^{1} \&$ Étienne Lengline ${ }^{2} \&$ Cora Knol-Bout $^{3} \&$ Paul Bosman $^{3}$ \& Dirk-Jan Eikema ${ }^{3}$ \& Boris Afanasyev ${ }^{4} \&$

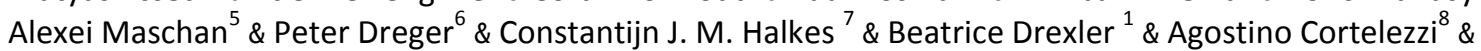 \\ Bernard Drénou ${ }^{9} \&$ Andrea Patriarca ${ }^{10}$ \& Benedetto Bruno ${ }^{11}$ \& Daniela Onofrillo ${ }^{12}$ \& Edoardo Lanino ${ }^{13}$ \& \\ Drazen Pulanic ${ }^{14}$ \& Ranka Serventi-Seiwerth ${ }^{14}$ \& Alice Garnier ${ }^{15}$ \& Per Ljungman ${ }^{16}$ \& Francesca Bonifazi ${ }^{17}$ \& \\ Sabrina Giammarco ${ }^{18}$ \& Olivier Tournilhac ${ }^{19}$ \& Pietro Pioltelli $^{20}$ \& Alicia Rovó ${ }^{21}$ \& Antonio M. Risitano ${ }^{22}$ \& \\ Régis Peffault de Latour ${ }^{2} \&$ Carlo Dufour ${ }^{13}$ \& Jakob Passweg ${ }^{1} \&$ for the EBMT SAA Working Party
}

\section{Abstract}

Eltrombopag (ELT), an oral thrombopoietin receptor agonist, has recently emerged as a promising new drug for the treatment of aplastic anemia (AA). How ELT is used outside of clinical trials in the real-world setting and results of this treatment are not known. We conducted therefore a retrospective survey on the use of ELT in AA among EBMT member centers. We analyzed the 134 patients reported in our survey together with 46 patients recently published by Lengline et al. The median follow-up from start of ELT treatment was 15.3 months, with $85.6 \%$ patients alive at last follow-up. Importantly, only $28.9 \%$ of our patients received ELT according to the FDA/EMA label as monotherapy in the relapsed/refractory setting, whereas $16.7 \%$ received ELT upfront. The overall response rate in our cohort was $62 \%$, very similar to the results of the pivotal ELT trial. In multivariate analysis, combination therapy with ELT/cyclosporine/ATG and response to previous therapy were associated with response. Overall survival was favorable with a 1-year survival from ELT start of 87.4\%. We identified age, AA severity before ELT start and response to ELT as variables significantly associated with OS. Two patients transformed to MDS; other adverse events were mostly benign. In sum, ELT is used widely in Europe to treat AA patients, mostly in the relapsed/refractory setting. Response to

1 Department of Hematology, University HospitalBasel,Petersgraben 4, 4031 Basel, Switzerland

2 Hopital St. Louis, Paris, France

3 EBMT Data Office, Leiden, Netherlands

4 First State Pavlov Medical University of St. Petersburg, St. Petersburg, Russia

5 Dimitri Rogachev Federal Research Center for Pediatric Hematology, Oncology and Immunology of Russian Federation, Moscow, Russia

6 University of Heidelberg, Heidelberg, Germany

7 Leiden University Hospital, Leiden, Netherlands

8 Fondazione IRCCS Ca' Granda Ospedale Maggiore Policlinico, and University of Milan, Milan, Italy

9 Hôpital Emile Muller, Mulhouse, France

${ }^{10}$ Division of Hematology, Department of Translational Medicine, University of Eastern Piedmont, Novara, Italy
${ }^{11}$ S.S.C.V.D Trapianto di Cellule Staminali, Torino, Italy

${ }^{12}$ Ospedale Civile, Pescara, Italy

${ }^{13}$ Institute Giannina Gaslini, Genoa, Italy

${ }^{14}$ University Hospital Center and Medical School, University of Zagreb, Zagreb, Croatia

${ }^{15}$ Clinical Hematology, CHU Nantes, Nantes, France

${ }^{16}$ Karolinska University Hospital, Stockholm, Sweden

${ }^{17}$ S.Orsola-Malpighi Hospital, Bologna University, Bologna, Italy

${ }^{18}$ Fondazione Policlinico Universitario A. Gemelli-IRCCS, Rome, Italy

${ }^{19}$ CHU ESTAING, Clermont, France

${ }^{20}$ Ospedale San Gerardo, Monza, Italy

${ }^{21}$ Department of Hematology and Central Hematology Laboratory, Inselspital, Bern University Hospital, University of Bern, Bern, Switzerland

${ }^{22}$ Hematology, Department of Clinical Medicine and Surgery, Federico II University of Naples, Naples, Italy 
ELT is similar to the clinical trial data across different age groups, treatment lines, and treatment combinations and results in favorable survival.

Keywords Aplastic anemia $\cdot$ Eltrombopag $\cdot$ Growth factors $\cdot$ Immunosuppression

\section{Introduction}

Severe aplastic anemia (SAA) is a rare heterogenous disease mostly autoimmune, in which T cell attack on hematopoietic stem (HSCs) and progenitor cells results in progressive bone marrow failure [1]. In patients not selected for potentially curative allogeneic hematopoietic stem cell transplantation (alloHSCT), the standard therapy consists of immunosuppression (IS) with horse anti-thymocyte globulin (ATG) and cyclosporine A (CYA) [2]. About $70 \%$ of patients do respond, but survival with good marrow function without relapse is in the order of only $30-40 \%$ [3]. Treatment options are unsatisfactory for patients refractory to or relapsing after first-line treatment and not eligible for allo-HSCT from a matched unrelated donor, with response rates to an additional course of ATG or anabolic steroids in the order of 30-60\% [4-6]. In addition to the modest efficacy, repeated courses of iimunosuppression (IS) may predispose to secondary malignancies [7]. Furthermore, the intensive immunosuppressive ATG-CYA combination cannot be applied in a proportion of AA patients due to advanced age or contraindications such as active infection [8].

Eltrombopag (ELT), a small molecule thrombopoietin (TPO) mimetic has been introduced in the AA therapy armamentarium recently. In contrast to previous failed attempts to stimulate the HSC compartment by diverse growth factors such as G-CSF, GM-CSF, or EPO [3, 9], ELT induced trilinear hematopoietic responses in refractory SAA patients [10]. Mechanistically, ELT binds to the TPO receptor c-MPL expressed on HSCs and leads to their proliferation and expansion [11,12], although additional, immune modulatory effects might also be involved [13]. Results of a phase II trial examining ELT monotherapy in refractory SAA patients showing a $40 \%$ response rate $[10,14]$ led to the approval of ELT as monotherapy in relapsed-refractory SAA in the USA and Europe $[15,16]$. Recently, increased response rate to ELT in combination with standard ATG-CYAimmunosuppression as compared to historical controls has been reported by the NIH group [17] and the use of ELT in the first-line setting is currently being tested in several ongoing clinical trials. Less is known about the use of ELT outside clinical trials. So far, realworld data of two cohorts described robust response to ELT administered as monotherapy or part of various combination regimens [18-20]. Romiplostim is a peptide-analogue TPO agonist with a very different chemical structure and different binding site on MPL, but similar efficacy as ELT in immune thrombocytopenia(ITP) [21]. Although romiplostim has been shown also to stimulate HSCs in animal models [11], its use in SAA remains anecdotal [19]. As in ITP switch from one TPO agonist to the other in refractory patients results in a high response rate [22], exploration of romiplostim for the treatment of SAA patients is clearly warranted.

Although the first results on ELT in SAA are promising, its optimal use, the necessity for concomitant IS, as well as predictors of response or incidence of relapse remain unknown, particularly for patients not representative of the clinical trial population or treated outside of the FDA/EMA label. Similarly, some safety concerns about clonal evolution and transformation to myelodysplastic syndromes in patients on ELT remain [14]. Analysis of a large cohort of SAA patients treated with ELT outside clinical trials is likely to give valuable insight into these open questions. Therefore, the EBMT SAA working party conducted a Europe-wide survey on the use of TPO agonists to treat AA in member centers. Here we report the results of the largest cohort of ELT treated AA patients so far.

\section{Methods}

\section{Data collection}

The EBMT SAA working party invited 441 EBMT centers to participate in this survey, $103(23 \%)$ centers answered, 92 $(21 \%)$ accepted to participate. Fifty centers $(11 \%)$ with potentially eligible patients received the survey form (supplementary material) based on the EBMT minimal-essential data (MED) $\mathrm{B}+\mathrm{C}$ forms used to record SAA patients in the EBMT registry. Patients with AA (moderate, severe, very severe) or PNH with AA phenotype treated with TPO agonists (ELT or romiplostim) either as first-line treatment or as rescue treatment outside clinical study protocols could be included in the survey. We considered ELT as first-line treatment if it was started within 60 days from beginning of the first therapy. Rescue treatment includes patients refractory or with insufficient response to one or several initial line(s) of therapy. Relapse includes patients who lost their response to any previous therapy, including those failing initial IS therapy for relapse. 
Complete response (CR) was defined as recovery of blood counts (hemoglobin $>100 \mathrm{~g} / \mathrm{l}$, neutrophil count $>1.5 \times 10^{6} \%$ $\mathrm{ml}$, platelet count $\left.>100 \times 10^{6} / \mathrm{ml}\right)$. Partial response $(\mathrm{PR})$ was defined as transfusion independence with any degree of improvement of blood counts. Minimal response was defined as some improvement in one or more lineage but not fulfilling the criteria of PR. No response refers to patients not fulfilling any of the above criteria.

We received data on 137 patients from 31 EBMT centers. In addition, we obtained individual patient level data from the French Reference Center for Aplastic Anemia on ELT-treated SAA patients published recently [20]. As the two cohorts had very similar inclusion/exclusion criteria, the two cohorts were merged in a single database (total 183 patients from 46 centers, median number of patients per center 2, range 1-21).

\section{Data analysis}

Due to low numbers $(n=3)$, patients treated with romiplostim were not considered further. Analyses were conducted on all ELT treated patients $(n=180)$.

Descriptive statistics are presented as percent of all patients with available data. Categorical data were compared using the Chi-square test, continuous variables were analyzed using the Kruskal-Wallis non-parametric test. Survival is presented using the Kaplan-Meier estimator. Comparisons among groups were by the log-rank test, multivariate models were created using the Cox regression model including response to ELT as a timedependent covariate. Response to ELT was analyzed as cumulative incidence with death as a competing event. Univariate comparisons were done using the Gray test and multivariate models were built according to Fine and Gray [23] using EZR software [24]. A $p$ value of $<0.05$ was considered significant.

\section{Results}

\section{Use of ELT for AA in Europe}

Treatment episodes started from 11/2011 to 10/2017. The median follow-up from start of ELT treatment was 15.3 months (95\% CI 13-17.6 months), with $85.6 \%$ patients alive at last follow-up. All patients were treated with ELT, in a median and maximum dose of $150 \mathrm{mg} /$ day, 11 patients $(6.4 \%)$ received a higher dose up to $450 \mathrm{mg}$. ELT dose was not different in patients with or without a response. ELT was ongoing in $50 \%$ of patients alive at last follow-up.

A minority of patients ( $n=30,16.7 \%)$ received ELT upfront, i.e., within 60 days following initiation of first-line treatment, whereas most patients were treated with ELT as a rescuetreatment either for refractory disease or relapse (Fig. 1a). ELT was applied both as monotherapy and in combination with IS with $\mathrm{CYA} \pm$ ATG (Fig. 1b). Based on this information, different (a) Treatment line

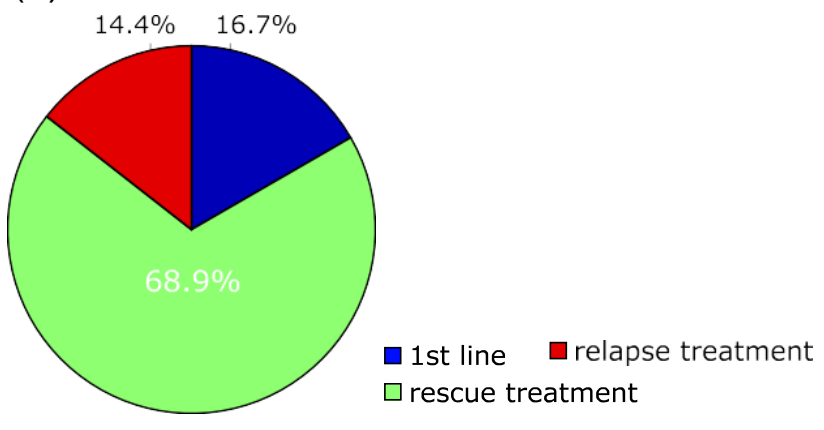

(b) ELT combinations

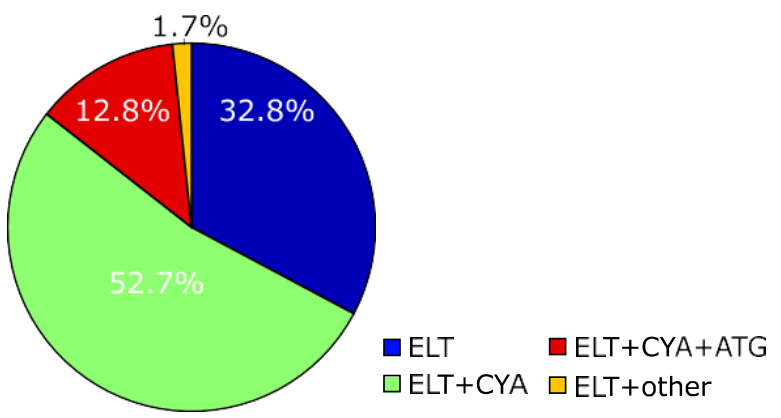

(c) Treatment patterns

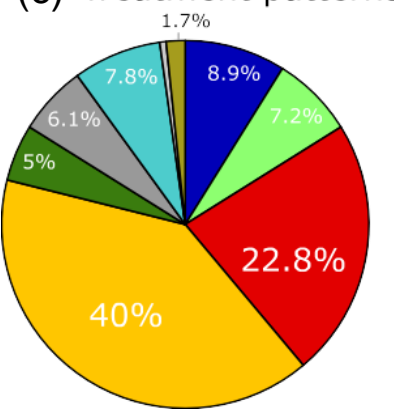

1st line, ELT, ELT/CYA aRescue, ELT/CYA a Relapse, ELT/CYA

$\square$ 1st line, ELT/CYA/ATG $\square$ Rescue, ELT/CYA/ATG aRelapse, ELT/CYA/ATG aRescue, ELT

口Relapse, ELT

$\square$ other

Fig. 1 Patterns of ELT use. a Treatment line, b combination regimens, and $\mathrm{c}$ treatment groups

associations with ELT are represented in our study population (Fig. 1c). Importantly, $71.1 \%$ of patients received ELT outside the FDA/EMA label, either in the first-line setting or as part of a combination therapy (irrespective of the therapy line). There was a significant $(p<0.001)$ association between treatment line and ELT combination, as the proportion of patients treated with ELT alone increased form first-line treatment to relapse, whereas ELT-CYA-ATG was used predominantly in the first-line setting (Table 1). Overall, 23 (12.8\%) patients received ELT in combination with CYA-ATG (within 60 days of 1 st ATG day), starting at day 14 (median) from the first ATG dose. 
Table 1 ELT treatment combinations according to treatment line

\begin{tabular}{llll}
\hline$p<0.001$ & \multicolumn{2}{l}{ Treatment line } & \\
\cline { 2 - 4 } ELT combination & $\begin{array}{l}\text { First-line } \\
n=30\end{array}$ & $\begin{array}{l}\text { Rescue treatment } \\
n=124\end{array}$ & $\begin{array}{l}\text { Relapse treatment } \\
n=26\end{array}$ \\
\hline ELT & $23.3 \%$ & $33.1 \%$ & $42.3 \%$ \\
ELT+CyA & $30 \%$ & $58.1 \%$ & $53.8 \%$ \\
ELT+CyA/ATG & $43.3 \%$ & $7.3 \%$ & $3.8 \%$ \\
ELT+other & $3 \%$ & $1.6 \%$ & $0 \%$ \\
& $100 \%$ & $100 \%$ & $100 \%$ \\
\hline
\end{tabular}

$p$ value from chi-square test

\section{Baseline characteristics}

Baseline characteristics of the whole cohort are shown in Table 2. Most patients were dependent on RBC and PLT transfusions (93.3\% and 90\%, respectively) preceding ELT therapy. Interestingly, the choice of the ELT treatment combination (monotherapy vs. combination therapy) can be at least partly explained by differences in age or AA severity, as patients treated with ELT monotherapy were older and were more likely to present with moderate AA at diagnosis compared to patients treated with combination therapy $(p=<001$ and $p=0.069$, respectively, supplementary Table 1 ).

\section{Response}

The reported overall response rate in our cohort was $62 \%$, with $17.9 \% \mathrm{CR}, 26.8 \% \mathrm{PR}$, and $17.3 \%$ minimal response. In patients responding, best response was achieved in median after 224 days (95\% confidence interval 136.5-311.5 days) (Fig. 2), although responses were reported at considerably later time points (range 8-1335 days). Time to first response was likely shorter, as response depth likely increases over time, but this information was not assessed in the survey. Twenty-fourpatients (21.6\% of responding) stoppedELTafter

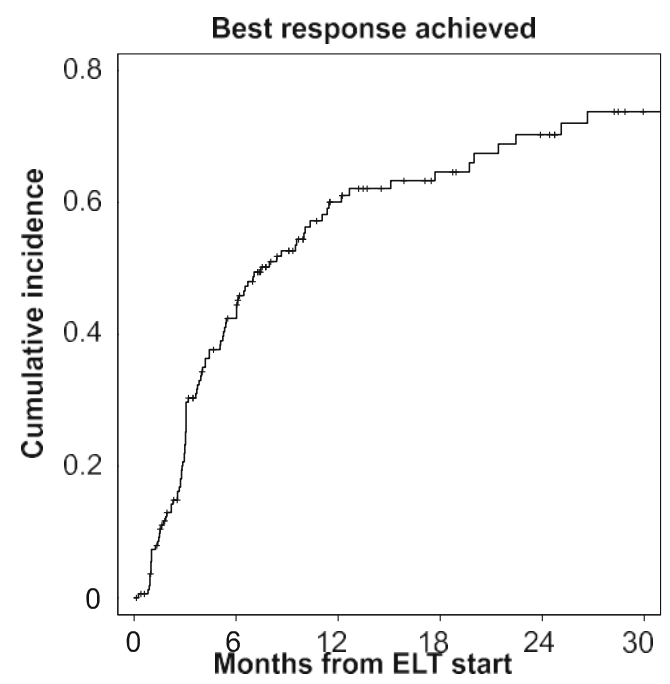

Fig. 2 Time to best response

having achieved a stable response, four $(16.7 \%)$ relapsed in median 83 days (range 53-980) following response and in median 246 days (range 164-988 days) after start of ELT.

Overall, relapse occurred in 15 patients (14.7\% of patients responding), in median 181 days (95\% CI 162.7-199.3 days) after start of ELT. The median duration of response was not reached.

The response rate did not differ between treatment combinations $(p=0.774)$, treatment line $(p=0.33)$ or according to treatment groups shown in Fig. 1c $(p=0.699)$. We analyzed thus our cohort as a whole and sought to identify factors predicting response in a time-to-event manner.

In univariate models, we could not identify any baseline characteristic associated with response in the whole cohort, although severity of AA at diagnosis and at the time of ELT start, as well as first-line treatment were significantly associated with death without a response (Tables 3 and 4, Fig. S1). In the multivariate model, combination therapy was a predictor $(p=0.04)$ of response, with a hazard ratio for response of 2.95 (95\% CI 1.28-6.83, $p=0.01$ ) for the ELT/CYA/ATG
Table 2 Baseline characteristics

\begin{tabular}{|c|c|c|c|}
\hline Sex & & Age at start ELT, years, median (IQR) & $48.4(23.3-67)$ \\
\hline Male & $56.1 \%$ & Severity of AA at start ELT & \\
\hline Female & $43.9 \%$ & Moderate & $16.8 \%$ \\
\hline $\begin{array}{l}\text { Age at diagnosis, years, } \\
\text { median (IQR) }\end{array}$ & $43.4(20-65.5)$ & Severe & $64.7 \%$ \\
\hline Severity of AAat diagnosis & & Very severe & $17.9 \%$ \\
\hline Moderate & $13.5 \%$ & $\begin{array}{l}\text { Number of previous treatment lines, } \\
\text { median (IQR) }\end{array}$ & $1(1-2)$ \\
\hline Severe & $55.6 \%$ & $\begin{array}{l}\text { Days from diagnosis to ELT start, } \\
\text { median (IQR) }\end{array}$ & $348.5(195-1212)$ \\
\hline Very severe & $30.4 \%$ & $\begin{array}{l}\text { Days from last treatment to ELT start, } \\
\text { median (IQR) }\end{array}$ & $140(53-429)$ \\
\hline PNH clone & $18.9 \%$ & $\begin{array}{l}\text { Days from last ATG to ELT start, } \\
\text { median (IQR) }\end{array}$ & $14(7-20.5)$ \\
\hline
\end{tabular}


Table 3 Univariate analysis of response with death as a competing event

\begin{tabular}{|c|c|c|c|c|c|c|c|}
\hline \multirow[t]{2}{*}{ Event: } & \multirow[t]{2}{*}{$n=$} & \multicolumn{3}{|l|}{ Response } & \multicolumn{3}{|l|}{ Death } \\
\hline & & 1-year incidence & $95 \% \mathrm{CI}$ & $p$ value & 1-year incidence & $95 \% \mathrm{CI}$ & $p$ value \\
\hline \multicolumn{8}{|l|}{ All patients } \\
\hline Combination & & & & 0.15 & & & 0.163 \\
\hline Monotherapy & 50 & 0.47 & $0.31-0.61$ & & 0.12 & $0.04-0.24$ & \\
\hline $\mathrm{ELT}+\mathrm{Cya}$ & 91 & 0.61 & $0.5-0.71$ & & 0.05 & $0.02-0.11$ & \\
\hline $\mathrm{ELT}+\mathrm{CyA} / \mathrm{ATG}$ & 23 & 0.66 & $0.4-0.83$ & & 0.15 & $0.03-0.36$ & \\
\hline \multicolumn{8}{|l|}{ Treatment line } \\
\hline First-line & 26 & 0.48 & $0.26-0.67$ & 0.43 & 0.26 & $0.1-0.47$ & $<0.001$ \\
\hline Rescue treatment & 116 & 0.59 & $0.49-0.68$ & & 0.05 & $0.02-0.11$ & \\
\hline Relapse treatment & 25 & 0.56 & $0.32-0.73$ & & 0.05 & $0.003-0.22$ & \\
\hline \multicolumn{8}{|l|}{ Sex } \\
\hline Male & 93 & 0.55 & $0.43-0.64$ & 0.29 & 0.08 & $0.03-0.15$ & 0.77 \\
\hline Female & 74 & 0.6 & $0.47-0.71$ & & 0.09 & $0.04-0.17$ & \\
\hline AA severity at diagnosis & & & & 0.5 & & & $<0.001$ \\
\hline Moderate & 23 & 0.59 & $0.35-0.77$ & & 0.04 & $0.003-0.19$ & \\
\hline Severe & 88 & 0.57 & $0.46-0.68$ & & 0.03 & $0.01-0.08$ & \\
\hline Very severe & 47 & 0.55 & $0.37-0.69$ & & 0.21 & $0.1-0.36$ & \\
\hline \multicolumn{8}{|l|}{ AA severity at ELT start } \\
\hline Moderate & 28 & 0.66 & $0.44-0.81$ & 0.42 & 0 & & $<0.001$ \\
\hline Severe & 106 & 0.58 & $0.47-0.67$ & & 0.03 & $0.01-0.1$ & \\
\hline Very severe & 27 & 0.4 & $0.21-0.59$ & & 0.38 & $0.16-0.6$ & \\
\hline \multicolumn{8}{|l|}{ Etiology } \\
\hline AA & 137 & 0.58 & $0.48-0.66$ & 0.9 & 0.09 & $0.05-0.15$ & 0.5 \\
\hline $\mathrm{PNH}$ & 30 & 0.56 & $0.34-0.72$ & & 0.04 & $0.002-0.17$ & \\
\hline \multicolumn{8}{|l|}{ Patients with previous treatment } \\
\hline ATG-exposed & & & & 0.91 & & & 0.84 \\
\hline No & 41 & 0.58 & $0.39-0.74$ & & 0.03 & $0.002-0.12$ & \\
\hline Yes & 100 & 0.59 & $0.48-0.68$ & & 0.06 & $0.02-0.12$ & \\
\hline Response to previous therapy & & & & 0.02 & & & 0.34 \\
\hline No & 98 & 0.533 & $0.42-0.63$ & & 0.06 & $0.02-0.13$ & \\
\hline Yes & 34 & 0.722 & $0.53-0.85$ & & 0.031 & $0.002-0.14$ & \\
\hline
\end{tabular}

group compared to ELT monotherapy (Tables 3 and 4). In the subgroup of patients with previous therapy, response to previous therapy was a predictor of response to subsequent ELT treatment (HR 1.689, 95\% CI 1.04-2.75, $p=0.04$ ), whereas previous exposure to ATG, interval from diagnosis or interval from last treatment were not significantly associated with response (Tables 3 and 4).

\section{Survival}

The median survival was not reached, the 1-year survival from start of ELTwas $87.4 \%$ (95\% CI 81.9-92.9\%) (Fig. 3a). Of the 26 patients who died during follow-up, seven $(26.9 \%)$ had undergone allo-HSCT and in six of these, deaths were classified as HSCT-related. In univariate and multivariate analysis, age at ELT start, and AA severity before ELT start were significantly associated with OS. Response to ELT included as a time-dependent covariate was similarly correlated with a lower incidence of death (Tables 5 and 6, Fig. 3b).

\section{Safety}

Adverse events were reported in 51 patients, although severe (grade III-IV) AEs were rare (17 patients, 30.4\% of all AEs). The most frequent AE was hepatic toxicity, a well-known side effect of ELT (Table 7). Skin reactions were rare. Importantly, ELT was stopped only in four patients due to AEs. 
Table 4 Multivariate analysis of response with death as a competing event

\begin{tabular}{|c|c|c|c|}
\hline & HR for response & $95 \% \mathrm{CI}$ & $p$ value \\
\hline \multicolumn{4}{|l|}{ All patients ( $n=155$ evaluable) } \\
\hline Combination & & & Overall (Wald test):0.04 \\
\hline Monotherapy & 1 (reference) & & \\
\hline $\mathrm{ELT}+\mathrm{CYA}$ & 1.36 & $0.77-2.4$ & 0.29 \\
\hline $\mathrm{ELT}+\mathrm{CYA} / \mathrm{ATG}$ & 2.95 & $1.28-6.83$ & 0.01 \\
\hline Treatment line & & & Overall (Wald test): 0.16 \\
\hline First-line & 1 (reference) & & \\
\hline Rescue treatment & 1.96 & $0.91-4.2$ & \\
\hline Relapse treatment & 2.51 & $0.94-6.7$ & \\
\hline \multicolumn{4}{|l|}{ Sex } \\
\hline Male & 1 (reference) & & \\
\hline Female & 1.08 & $0.69-1.67$ & \\
\hline AA severity at diagnosis & & & Overall (Wald test):0.72 \\
\hline Moderate & 1 (reference) & & \\
\hline Severe & 1.46 & $0.59-3.61$ & \\
\hline Very severe & 1.47 & $0.51-4.2$ & \\
\hline AA severity at ELT start & & & Overall (Wald test): 0.52 \\
\hline Moderate & 1 (reference) & & \\
\hline Severe & 0.67 & $0.3-1.49$ & \\
\hline Very severe & 0.54 & $0.1842-1.59$ & \\
\hline \multicolumn{4}{|l|}{ Etiology } \\
\hline AA & 1 (reference) & & \\
\hline $\mathrm{PNH}$ & 0.78 & $0.41-1.45$ & \\
\hline Age at ELT start & 1.01 & $1.0-1.02$ & \\
\hline \multicolumn{4}{|c|}{ Patients with previous treatment ( $n=123$ evaluable) } \\
\hline \multicolumn{4}{|l|}{ ATG-exposed } \\
\hline No & 1 (reference) & & \\
\hline Yes & 0.853 & $0.46-1.57$ & 0.61 \\
\hline \multicolumn{4}{|l|}{ Response to previous therapy } \\
\hline No & 1 (reference) & & \\
\hline Yes & 1.689 & $1.04-2.75$ & 0.035 \\
\hline Time from diagnosis to ELT start & 0.9998 & $0.9995-1$ & 0.28 \\
\hline Time from last treatment to ELT start & 1 & $0.9999-1$ & 0.16 \\
\hline
\end{tabular}

Cytogenetic abnormalities were assessed at diagnosis and showed a normal karyotype in 123 patients (91.1\%) and an abnormal karyotype in 12 cases (8.9\%) including 3 cases of trisomy 8, 2 cases of monosomy 7, and 1 case of inv. (3;3q26). Cytogenetic information was not available for the remaining cases $(n=45,25 \%$ of the whole cohort). Cytogenetic evolution during the course of the treatment was not assessed in the survey. Only two cases of transformation into myelodysplastic syndrome (MDS) were described, diagnosed 2 and 5.5 months after ELT start. One of these two patients with a subsequent MDS transformation had a monosomy $\mathrm{X}$ at AA diagnosis, the other had no cytogenetic information available. No other secondary malignancies apart from two cases of squamous cell carcinoma of the skin occurred.

\section{Discussion}

Whereas IS addresses the autoimmune destruction of HSCs, allo-HSCT has been so far the only treatment modality correcting also the resulting stem cell defect in AA patients. The efficacy of ELT as monotherapy, presumably acting preferentially at the stem cell level, created much excitement in the field. The next logical step, combining ELT with IS has been 

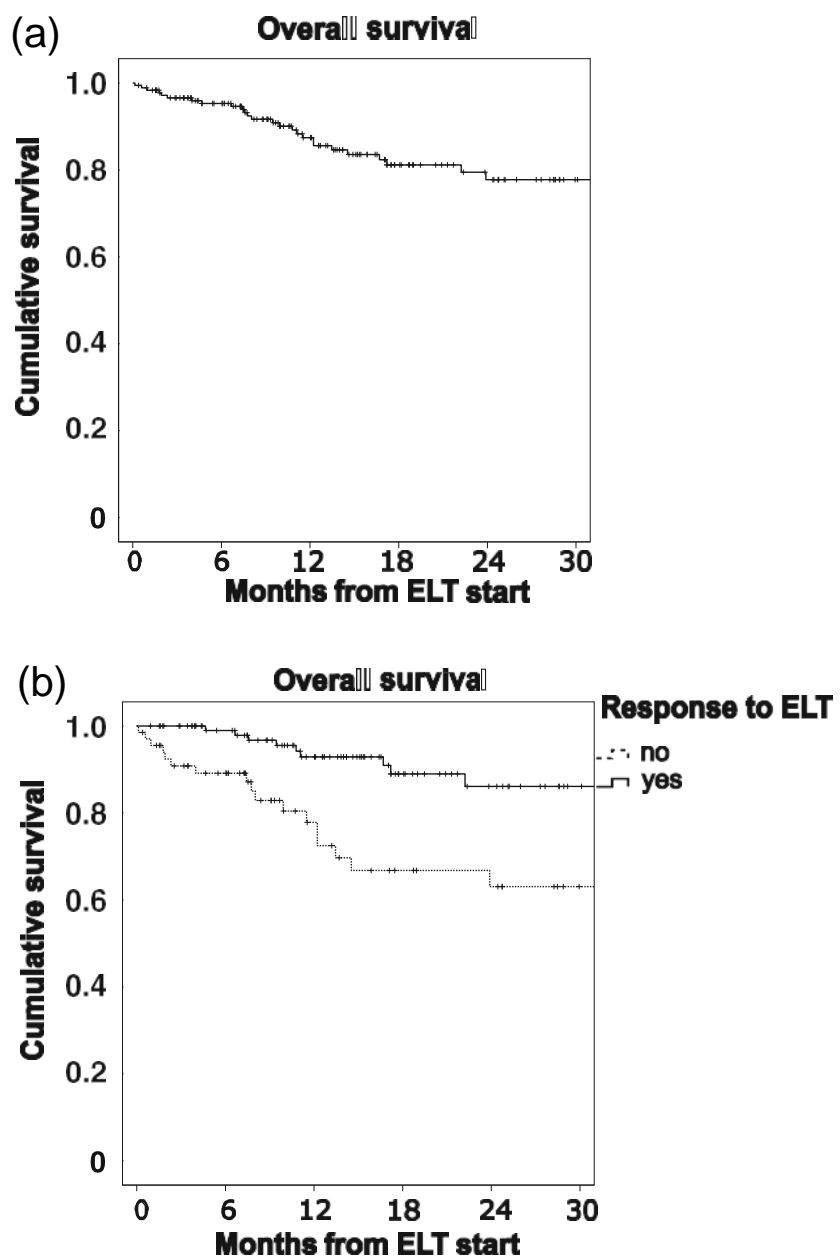

Fig. 3 Overall survival. a Whole cohort, b according to response to ELT, please refer also to the multivariate analysis of survival considering response to ELT as a time-dependent covariate

explored in phase II trials $[17,25]$ and is being examined in several ongoing phase III trials. Until the role of ELT in the first-line setting is clearly defined, ELT will be used mostly in patients relapsed or refractory to IS or allo-HSCT.

Table 5 Univariate analysis of survival

\begin{tabular}{ll}
\hline Univariate analysis for OS (log-rank test) & \\
Variable & $p$ value \\
\hline Sex & 0.62 \\
AA severity at diagnosis & 0.17 \\
Age at start ELT & 0.01 \\
PNH & 0.11 \\
AA severity at ELT start & $<0.001$ \\
ELT combination & 0.55 \\
Treatment line & 0.1 \\
Prev. ATG exposure & 0.92 \\
Best response to previous treatment & 0.65 \\
\hline
\end{tabular}

Table 6 Multivariate analysis of survival

\begin{tabular}{lllll}
\hline \multicolumn{2}{l}{ Multivariate analysis for OS } & & & \\
\hline Variable & $p$ value & HR for death & 0.95 CI & \\
\hline Age at start ELT & 0.01 & 1.026 & 1.005 & 1.046 \\
AA severity at ELT start & 0 & & & \\
$\quad \begin{array}{llll}\text { Moderate } \\
\text { Severe }\end{array}$ & 0.49 & 1.7 & 0.4 & 7.9 \\
$\quad$ Very severe & 0.001 & 13.2 & 2.7 & 64 \\
ELT combination & 0.56 & & & \\
ELT & & & & \\
ELT+CYA & & & & \\
ELT+CYA/ATG & & & & \\
Response to ELT & 0.01 & & 0 & 0.7 \\
$\quad$ Yes (compared to none) & & 0.11 & & \\
\hline
\end{tabular}

In line with this, only a small minority in our cohort was treated upfront with an ELT containing regimen. Although the reasons for ELT application in the first-line setting were not evident, age and comorbidities precluding use of standard ATG/CYA therapy leading to ELT use as part of a low intensity therapy on one hand and high-risk AA resulting in ELT in combination with ATG/CYA on the other are likely explanations. Supporting this hypothesis, the median age of patients treated with ELT alone or with CYA in the first-line setting was 55.5 years, compared to 23 years $(p<0.001)$ in patients treated with ELT/CYA/ATG. Severity of AA at diagnosis was similar. Since this is likely a selection of patients and is not representative of the entire patient population, the role of ELT in the first-line setting could not be clarified in our cohort. The response rate of $46.7 \%$ in patients with low intensity ELT treatment in the first-line setting is certainly meaningful, but not above the range expected, e.g., with CYA alone [26].

In our cohort, relapsed/refractory patients were treated with ELT mostly in combination with CYA \pm ATG, unlike in the pivotal trial using ELT as a monotherapy [14]. Although failure of IS argues against retreatment with another course of IS

Table 7 Adverse events

\begin{tabular}{llll}
\hline Adverse events & & & \\
\hline & Grades I-II & Grades III-IV & Any grade \\
\hline Bleeding & $10.3 \%$ & $18.8 \%$ & $12.7 \%$ \\
Infection & $5.1 \%$ & $31.3 \%$ & $12.7 \%$ \\
Hepatic toxicity & $64.1 \%$ & $25.0 \%$ & $52.7 \%$ \\
Skin & $0.0 \%$ & $12.5 \%$ & $3.6 \%$ \\
Other & $20.5 \%$ & $18.8 \%$ & $18.2 \%$ \\
$n=$ (events) & 39 & 17 & 56 \\
\hline
\end{tabular}

The number and percentage of AEs corresponds to the events reported 
with ATG from as different source, alemtuzumab or an alternative calcineurin inhibitor, this strategy results in a response rate up to $50-80 \%$ [6, 27]. A detailed analysis of therapies preceding ELT was beyond the scope of our survey; we were therefore unable to discern whether patients benefitted from continuation of IS after insufficient response or retreatment with CYA/- ATG in relapse. Interestingly, a response to previous IS was a predictor of response to ELT only in patients treated with ELT in combination with IS, but not with ELT monotherapy. This might support independent predictive factors for response between IS and ELT, although differences in the number of patients in the two groups provides an alternative explanation. For CYA/ATG, several biological predictors of response, such as absolute reticulocyte count reflecting stem cell reserve or lymphocyte count and presence of a PNH clone reflecting the level of the immune attack have been defined $[28,29]$. Such variables were not systematically queried in our survey, but clinical parameters might provide an approximation of these predictors. Age and time from diagnosis to ELT treatment, as proxy for stem cell reserve, were not negatively correlated with response. Similarly, presence of a PNH clone or treatment of relapse following IS, arguing for a predominant autoimmune etiology, did not affect response rates. Finally, severity of AA at ELT start could also not predict response, although it did correlate with death without response in competing risk analysis. It is unlikely, that merely increasing the size of such retrospective cohorts can discern predictors of response. Additional mechanistic studies in preclinical models as well as correlative analyses in uniformly treated patients in clinical trials will answer these urgent questions. Compared to the response used in current clinical trials, our more traditional response criteria did not assess trilineage response. Since almost all patients were RBC transfusion dependent, PR or CR to ELT essentially implies an erythroid response and rules out an isolated increase of platelets upon ELT treatment. In our multivariate analysis, combination therapy compared to ELT monotherapy was associated with a better response; this difference was driven mainly by the better response in the ATG combination group. Multivariate analyses can control for some confounding factors; these results must be interpreted cautiously in light of the very different patient characteristics.

Although the use of a second-line therapy such as ELT likely identifies patients with a less than average prognosis, the overall survival in our cohort was favorable. A considerable proportion of patients died due to allo-HSCT-related causes and not from progression of AA. Whereas traditionally AA patients have been treated in allo-HSCT centers, ELT might be also applied nowadays in less-specialized centers not included in our survey and thus introducing bias particularly in this regard. The association between response and survival is inherently biased particularly in retrospective studies. Despite this limitation, response to ELT considered as a time-dependent variable in the multivariate Cox model was significantly associated with better survival, underscoring the importance of response.

Retrospective analyses likely underreport low-grade AEs, but the large number of patients is useful to detect rare, severe AEs of novel drugs. AEs in our survey were consistent with the favorable safety profile in AA trials and ITP patients, with common hepatic toxicity and a few cases of skin reactions. Although cytogenetic evolution was not systematically assessed in our survey, the low rate of MDS diagnosis (incidence of $\sim 1$ in 120 years of follow-up) is a reassuring signal, particularly since $6.7 \%$ of all patients had a cytogenetic abnormality before ELT treatment.

Additionallimitations of ourstudy include those associated with retrospective cohorts such as selection bias, recall bias, incomplete data, and limited follow-up. Conversely, the cohort represents real-world data from different countries across Europe. This is particularly important in a rare disease such as SAA with very different treatment trajectories often underrepresented in clinical trials. Along the same lines, the majority of the patients in our cohort received ELT outside of the EMA/ FDA label and our data clearly supports this clinical practice.

Our survey is in line with previous, considerably smaller cohorts of AA patients treated with ELT. Gill et al. [19] reported initially a cohort of ten refractory AA patients from Hong Kong treated with ELT alone or in combination with CYA. The study was recently updated with a longer follow-up and inclusion of additional patients [18]. Interestingly, the response rate of ten patients in the expansion cohort receiving ELT in the first-line setting was $90 \%$ compared to $50 \%$ in refractory patients. The higher response was not explained by the use of ATG in in the first-line patients, as all first-line patients treated with ELT/CYA without ATGresponded. We could not confirm the higher response rate in patients treated upfront with ELT.

Similar results were observed in a group of 46 , mostly relapsed or refractory patients from France [20]. Transfusion independence was achieved in 36\% and 49\% in ATG naïve and ATG pretreated patients, respectively. These 46 patients are notably contained in our analysis. In our whole cohort, $47.6 \%$ achieved at least a PR and thus transfusion independence. In contrast to the French cohort, there was no difference in time to response in patients with a response in the firstline vs. rescue/relapse setting.

In summary, ELT is already widely used in Europe among EBMT centers mostly as treatment for insufficient response to previous therapies or as relapse treatment. In contrast to the available clinical trial data, ELT is applied mostly incombination with CYA. The response rate was consistent across all treatment situations and was similar across combinations. The results of ongoing clinical trials with ELT in the first-line setting, as well as mechanistic studies investigating synergism between ELT and IS are eagerly awaited. 
Acknowledgements We would like to thank all centers contributing patient data to the survey.

\section{Compliance with ethical standards}

All procedures performed were in accordance with the ethical standards of the applicable institutional and national research committees, with the 1964 Helsinki declaration and its later amendments as well as comparable ethical standards. All patients provided informed consent to inclusion of their data in the EBMT SAA registry.

\section{References}

1. Luzzatto L, Risitano AM (2018) Advances in understanding the pathogenesis of acquired aplastic anaemia. Br J Haematol 182(6): 758-776

2. Killick SB, Bown N, Cavenagh J, Dokal I, Foukaneli T, Hill A, Hillmen P, Ireland R, Kulasekararaj A, Mufti G, Snowden JA, Samarasinghe S, Wood A, Marsh JCW, the British Society for Standards in Haematology (2016) Guidelines for the diagnosis and management of adult aplastic anaemia. Br J Aematol 172(2): 187-207

3. Tichelli A, Schrezenmeier H, Socié G et al (2011) A randomized controlled study in patients with newly diagnosed severe aplastic anemia receiving antithymocyte globulin (ATG), cyclosporine, with or without G-CSF: a study of the SAA Working Party of the European Group for Blood and Marrow Transplantation. Blood 117(17):4434-4441

4. Scheinberg P, Nunez O, Young NS (2006) Retreatment with rabbit anti-thymocyte globulin and ciclosporin for patients with relapsed or refractory severe aplastic anaemia. Br J Haematol 133(6):622627

5. Giammarco S, van Lint M, Lamparelli Tetal Androgens may boost hematologic response to anti-thymocyte globulin in acquired aplastic anemia. Blood 128(22):3900

6. Tichelli A, Passweg J, Nissen C, Bargetzi M, Hoffmann T, WodnarFilipowicz A, Signer E, Speck B, Gratwohl A (1998) Repeated treatment with horse antilymphocyte globulin for severe aplastic anaemia. Br J Haematol 100(2):393-400

7. Socie G, Henry-Amar M, Bacigalupo A, Hows J, Tichelli A, Ljungman P, McCann SR, Frickhofen N, van't Veer-Korthof E, Gluckman E (1993) Malignant tumors occurring after treatment of aplastic anemia. N Engl J Med 329(16):1152-1157

8. Kao SY, Xu W, Brandwein JM, Lipton JH, Messner HA, Minden MD, Schimmer AD, Schuh AC, Yee K, Gupta V (2008) Outcomes of older patients ( $\geq 60$ years) with acquired aplastic anaemia treated with immunosuppressive therapy. Br J Haematol 143(5):738-743

9. Marsh J, Ganser A, Stadler M(2007) Hematopoietic growth factors in the treatment of acquired bone marrow failure states. Semin Hematol 44(3):138-147

10. Desmond R, Townsley DM, Dumitriu B et al (2014) Eltrombopag restores trilineage hematopoiesis in refractory severe aplastic anemia that can be sustained on discontinuation of drug. Blood. 123(12):1818-1825

11. Kovtonyuk LV, Manz MG, Takizawa H (2016) Enhanced thrombopoietin but not G-CSF receptor stimulation induces self- renewinghematopoietic stemcell divisions in vivo. Blood 127(25): 3175-3179

12. Sun H, Tsai Y, Nowak I, Liesveld J, Chen Y (2012) Eltrombopag, a thrombopoietin receptor agonist, enhances human umbilical cord blood hematopoietic stem/primitive progenitor cell expansion and promotes multi-lineage hematopoiesis. Stem Cell Res 9(2):77-86

13. Liu X, Liu S, Feng Q, Liu XN, Li GS, Sheng Z, Chen P, Liu Y, Wei Y, Dong XY, Qin P, Gao C, Ma C, Zhang L, Hou M, Peng J (2016) Thrombopoietin receptor agonists shift the balance of Fcyreceptors toward inhibitory receptor IIb on monocytes in ITP. Blood 128(6): 852-861

14. Olnes MJ, Scheinberg P, Calvo KR et al (2012) Eltrombopag and improved hematopoiesis in refractory aplastic anemia. N Engl J Med 367(1):11-19

15. Novartis. Revolade product information. July 2018. https://www. ema.europa.eu/documents/product-information/revolade-eparproduct-information_en.pdf. Accessed 9 Oct 2018

16. Novartis (2017) Promacta prescribing information. https://www. accessdata.fda.gov/drugsatfda_docs/label/2017/022291s019lbl. pdf. Accessed 9 Oct 2018

17. Townsley DM, Scheinberg P, Winkler T et al (2017) Eltrombopag added to standard immunosuppression for aplastic anemia. NEngl J Med 376(16):1540-1550

18. Hwang Y-Y, Gill H, Chan T, Leung G, Cheung C, Kwong Y-L (2018) Eltrombopag in the management of aplastic anaemia: real-world experience in a non-trial setting. Hematology 23(7): 399-404

19. Gill H, Leung GM, Lopes D, Kwong Y-L (2017) The thrombopoietin mimetics eltrombopag and romiplostim in the treatment of refractory aplastic anaemia. Br J Haematol 176(6): 991-994

20. Lengline E, Drenou B, Peterlin Pet al (2017) Nationwide survey on the use of eltrombopag in patients with severe aplastic anemia: a report on behalf of the French reference center for aplastic anemia. Haematologica 103(2):212-220

21. Rodeghiero F, Ruggeri M (2015) Treatment of immune thrombocytopenia in adults: the role of thrombopoietin-receptor agonists. Semin Hematol 52(1):16-24

22. Khellaf M, Viallard J-F, Hamidou M, Cheze S, Roudot-Thoraval F, Lefrere F, Fain O, Audia S, Abgrall JF, Michot JM, Dauriac C, Lefort S, Gyan E, Niault M, Durand JM, Languille L, Boutboul D, Bierling P, Michel M, Godeau B (2013) A retrospective pilot evaluation of switching thrombopoietic receptoragonists in immune thrombocytopenia. Haematologica 98(6): 881-887

23. Fine JP, Gray RJ (1999) A proportional hazards model for the subdistribution of a competing risk. J Am Stat Assoc 94(446): 496-509

24. Kanda Y (2013) Investigation of the freely available easy-to-use software 'EZR' for medical statistics. Bone Marrow Transplant 48(3):452-458

25. Assi R, Garcia-Manero G, Ravandi F, Borthakur G, Daver NG, Jabbour E, Burger J, Estrov Z, Dinardo CD, Alvarado Y, Hendrickson S, Ferrajoli A, Wierda W, Cortes J, Kantarjian H, Kadia TM (2018) Addition of eltrombopag to immunosuppressive therapy in patients with newly diagnosed aplastic anemia. Cancer 124(21):4192-4201

26. Gluckman E, Esperou-Bourdeau H, Baruchel A et al (1992) Multicenter randomized study comparing cyclosporine-A alone and antithymocyte globulin with prednisone for treatment of severe aplastic anemia. Blood 2540-2546 
27. Risitano A, Schrezenmeier H (2013) Alternative immunosuppression in patients failing immunosuppression with ATG who are not transplant candidates: Campath (Alemtuzumab). Bone Marrow Transplant 48:186-190

28. Scheinberg P, Wu CO, Nunez O, Young NS (2009) Predicting response to immunosuppressive therapy and survival in severe aplastic anaemia. Br J Haematol 144(2):206216
29. Sugimori C, Chuhjo T, Feng X et al (2006) Minor population of CD55-CD59-blood cells predicts response to immunosuppressive therapy and prognosis in patients with aplastic anemia. Blood 107(4):1308-1314 